\title{
A Reach, Effectiveness, Adoption, Implementation, Maintenance Evaluation of Weekend Backpack Food Assistance Programs
}

\section{Authors: Carmen Byker Shanks \& Samantha Harden}

This is a postprint of an article that originally appeared in American Joumal of tealth Promotion on September 2016. https://dx.doi.org/10.4278/ajhp.140116-QUAL-28

Shanks, Carmen Byker, and Samantha Harden. "A Reach, Effectiveness, Adoption, Implementation, Maintenance Evaluation of Weekend Backpack Food Assistance Programs." American Journal of Health Promotion 30, no. 7 (September 2016): 511-520. DOI: 10.4278/ ajhp.140116-QUAL-28. 


\title{
A Reach, Effectiveness, Adoption, Implementation, Maintenance Evaluation of Weekend Backpack Food Assistance Programs
}

\author{
Carmen Byker Shanks, PhD; Samantha Harden, PhD
}

\section{Abstract}

Purpose. The purpose of this study was to evaluate an ongoing statewide weekend backpack program through the Reach, Effectiveness, Adoption, Implementation, and Maintenance (RE-AIM) framework.

Design. Mixed-methods inquiry was used to explore the reach, effectiveness, adoption, implementation, and maintenance of backpack programs within Montana.

Setting. Study participants completed audio-recorded one-on-one phone interviews.

Participants. Key informants (e.g., managers at food banks, staff at participating schools, policy makers) were purposively sampled $(N=20)$.

Method. Semistructured interviews were conducted to gather data to describe each RE-AIM dimension. Interviews were transcribed verbatim and deductively (i.e., using RE-AIM as themes) coded for meaning units, placed into higher-order categories, and summarized in narrative. Supporting quantitative data (e.g., the proportion of eligible students that joined the program, rate of school-level adoption) were calculated using descriptive statistics.

Results. Backpack programs with a broad reach and evidenced effect may be appealing to adopt. Weekend food bags cost an average $\$ 3.87$ (SD \pm .94$)$ and there were some positive (i.e., ease, protecting participants' privacy) and very few negative (logistical) components of implementation. Collaborators and community partners are necessary for long-term sustainability.

Conclusion. Backpack programs are widespread and have potential to relieve weekend hunger; however, more efforts need to be made to end childhood hunger. (Am J Health Promot 0000;00[0]:000-000.)

Key Words: Food Security, RE-AIM, Program, Children, Prevention Research. Manuscript format: research; Research purpose: program evaluation; Study design: qualitative; Outcome measure: productivity; Setting: local community/state; Health focus: nutrition; Strategy: policy; Target population age: youth; Target population circumstances: income level

Carmen Byker Shanks, PhD, is with the Food and Health Lab in the Department of Health and Human Development, Montana State University, Bozeman, Montana. Samantha Harden, PhD, is with the Human Nutrition, Foods and Exercise Department, Virginia Tech, Blacksburg, Virginia.

Send reprint requests to Carmen Byker Shanks, PhD, Department of Health and Human Development, Montana State University, 222 Romney Gym, Bozeman, MT 59717; carmen.byker@ montana.edu.

This manuscript was submitted January 16, 2014; revisions were requested March 15, May 4, August 9, and November 11, 2014; the manuscript was accepted for publication January 25, 2015.

Copyright (C) 0000 by American Journal of Health Promotion, Inc.

0890-1171/00/\$5.00+0

DOI: 10.4278/ajhp.140116-QUAL-28

\section{PURPOSE}

Twenty percent of households in the United States with children were identified as food insecure in 2012 through the U.S. Department of Agriculture Economic Research Service annual food security survey. ${ }^{1}$

Among these households, $10 \%$ of children were also reported as food insecure. When children are hungry, their physical, ${ }^{2}$ mental, ${ }^{3}$ and behavioral and emotional health ${ }^{4}$ is compromised.

There are policy measures in place to help protect households with children from food insecurity in the United States. The recent reauthorization of the Healthy, Hunger-Free Kids Act of 2010 included legislation for the National School Lunch and Breakfast Programs, the Supplemental Nutrition Assistance Program, the Special Supplemental Nutrition Program for Women, Infants, and Children, the Child and Adult Care Food Program, and the Summer Food Service Program. ${ }^{5}$ These programs provide supplemental assistance to purchase food and provide nutrition education to recipients.

Beyond public assistance, communities also strategize several private ways to provide immediate food assistance though emergency food programs such as food banks, food pantries, and community feeding programs. ${ }^{6}$ For example, to solve the problem of children repeatedly arriving at school on Monday hungry as a result of their nutrient needs not being satisfied over the weekend, communities across the nation have implemented programs within schools where easy-to-prepare 
foods are distributed to students on Fridays to help reduce weekend hunger. ${ }^{7,8}$ Feeding America runs the largest program in the nation, the BackPack Program, serving 230,000 children each year and working through food banks in states across the United States. ${ }^{9}$

In Montana, $14.1 \%$ of households were identified as food insecure in 2012. ${ }^{1}$ Several efforts to increase food security for children exist in the state, including free or reduced-price $(F / R)$ breakfast and lunch programs, summer lunch programs, and the Montana Partnership to End Childhood Hunger (http:/ / mt.nokidhungry.org/ partnership-end-childhood-hunger).

Among these programs, the Montana Food Bank Network (MFBN) initiated the first Feeding America BackPack Program in 2008 at one school and the program has since grown statewide. ${ }^{10}$ To be considered a Montana Feeding America BackPack Program, the school must follow three standards: (1) approved agency in good standing with MFBN that complies with all applicable federal and local statutes, ordinances, and regulations; (2) healthy, nutritious food must be distributed to children free of charge; and (3) backpacks must be provided a minimum of once a month during the school year. ${ }^{10}$

Other food banks in Montana have also adopted the original model proposed by Feeding America, but operate independently of Feeding America and MFBN. During the 2011-2012 school year, the MFBN Feeding America BackPack Program served 45 K-12 schools through 17 food banks or pantries, and four other food banks served 25 other K-12 schools through a similar model (i.e., Kid Packs, Back Pack Program, Backpack for Kids, and Kids Pack Program) in Montana. BackPack and similar programs are referred to as backpack programs throughout the remainder of this paper.

The collective scope of the success and sustainability of the five backpack programs in Montana is unknown. Stakeholders in Montana identified that evaluation would assist in program development and a collective approach was taken to researching the combined function of backpack programs across the state. The Reach, Effectiveness, Adoption, Implementation, and Maintenance (RE-AIM) framework provides a guiding metric to better understand the ability of these programs to reach those who need food relief, the effectiveness of relieving weekend hunger, fidelity to the programmatic model, the rate of adoption, and sustainability of the weekend backpack food assistance program. ${ }^{11}$ The multifaceted dimensions of the RE-AIM framework capture internal and external validity factors in order to increase the generalizability of the program, policy, or practice. Although RE-AIM reviews on food security programs do not presently exist, we hypothesize that partnerships would influence the generalizability, program tailoring, and sustainability of a community food assistance program.

The purpose of this study was to (1) evaluate backpack programs across Montana to understand the collective public health impact of the emergency food program and (2) provide current and potential adopters with information to guide future practice. The results identify adaptations to the food security program to ensure setting-level program sustainability and individual access.

\section{DESIGN}

Overall, the methodology of this study aligns with a RE-AIM public health evaluation. As such, this primarily qualitative study utilized Glasgow and colleagues' 21-item measure ${ }^{12}$ to ensure the reporting on internal and external validity factors to enhance generalizability of research findings. Quantitative measures were utilized to support qualitative data. Guided by this 21-item measure, represented in Table 1 , quantitative or qualitative measures were developed to obtain information wherever applicable. For instance, the backpack programs provide anonymity for participants and collecting individual dietary data is not within protocol. As such, the entire dimension of effectiveness is based on intent-to-treat measures (e.g., Healthy Eating Index [HEI]-2010 is used to understand the nutrient quality and adequacy being delivered). ${ }^{13}$ Effectiveness of the back- pack program to deliver sufficient nutrients in other areas of the United States has been measured through HEI previously. ${ }^{14}$ HEI-2010 measures compliance with the 2010 Dietary Guidelines for Americans. ${ }^{13}$ HEI-2010 uses standards that are expressed as either a percentage of calories or per 1000 calories, separating diet quality from quantity. The sum of the scores for the food components is the total HEI-2010 score, which ranges from 0 to 100 , with a higher score indicative of a more healthful diet. In order to determine the nutrient value of a typical backpack, the investigators randomly selected one food bag (i.e., of those that would be delivered to the students) from each participating food bank. Food bag nutritional content was analyzed by entering food codes into Nutritionist Pro Diet Analysis Module (version 2.5; Axxya Systems, Stafford, Texas) and using HEI-2010 SAS code (version 9.2; SAS Institute Inc., Cary, North Carolina), provided by the USDA Center for Policy and Promotion. ${ }^{15}$ In addition to the HEI-2010, other items that determined the effect of the backpack program on relieving weekend hunger and reaching those most in need as well as information on program attrition were measured through interviews (reported in Table 1). Data was also collected through secondary sources, such as food bank program Web sites and manuals and Montana Office of Public Instruction public F/R meal eligibility data and school enrollment data. ${ }^{16-21}$ This study was conducted from July 2012 to August 2013, with key informant interviews and data collection occurring during the first 5 months and qualitative and quantitative analysis occurring during the remaining 7 months.

\section{SETTING}

The lead author interviewed key informants (e.g., school and food bank staff at all levels) during one-on-one audio-recorded telephone interviews to provide qualitative data based on the RE-AIM dimensions. The lead author took notes about points that were important to follow up about at a later time in the interview and key concepts that the participant discussed. 
Table 1

Items, Measures, and Sources for RE-AIM Dimensions in Montana-Based Weekend Backpack Food Assistance Program Evaluation During 2011-2012 School Year*

\begin{tabular}{|c|c|c|c|}
\hline Dimension & Items & Measure & Data Source \\
\hline \multirow[t]{5}{*}{ Reach } & $\begin{array}{l}\text { Method to identify target } \\
\text { population }\end{array}$ & How are children identified to participate in the program? & Interviews; program Web sites \\
\hline & Inclusion criteria & Process for programs to include children to participate? & Interviews; program Web sites \\
\hline & Exclusion criteria & Why would a child be excluded from program participation? & Interviews; program Web sites \\
\hline & Participation rate & No. food bags distributed/No. eligible children & Interviews; program Web sites \\
\hline & Representativeness & Does the program identify chronically hungry children? & Interviews; F/R eligibility data \\
\hline \multirow[t]{4}{*}{ Efficacy/effectiveness } & $\begin{array}{l}\text { Measures for at least one } \\
\text { follow-up }\end{array}$ & Nutrients provided in food bags compared to $\mathrm{HEI}-2010 \dagger$ & Food bag contents \\
\hline & Intent to treat & Are programs adequate in addressing childhood hunger? & Interviews \\
\hline & Quality of life & Results reported from stakeholders after implementation & Interviews \\
\hline & Percentage attrition & Do participants drop out of program? If so, why? & Interviews \\
\hline \multirow[t]{6}{*}{ Adoption } & $\begin{array}{l}\text { Description of intervention } \\
\text { location }\end{array}$ & $\begin{array}{l}\% \text { schools }>50 \% \mathrm{~F} / \mathrm{R} \text { eligible; location in Montana; } \\
\text { travel distance }\end{array}$ & F/R eligibility; interviews \\
\hline & Inclusion of setting & How are students chosen to participate? & Interviews; program Web sites \\
\hline & Exclusion of setting & Why would a school be excluded from program participation? & Interviews; program Web sites \\
\hline & Description of staff & Characteristics of staff who implement the program & Interviews \\
\hline & Level of expertise & Who implements the program? & Interviews \\
\hline & Adoption rate & No. schools enrolled/No. schools $>50 \%$ F/R eligible & $\begin{array}{l}\text { Enrollment; F/R eligibility; } \\
\text { interviews }\end{array}$ \\
\hline \multirow[t]{3}{*}{ Implementation } & $\begin{array}{l}\text { Type, frequency, and } \\
\text { intensity }\end{array}$ & Frequency of program delivery & Interviews \\
\hline & $\begin{array}{l}\text { Extent protocol delivered } \\
\text { as intended }\end{array}$ & Protocol for program distribution & Interviews \\
\hline & Measures of cost & Cost of program delivery by year by participation schools & Interviews \\
\hline \multirow[t]{3}{*}{$\begin{array}{l}\text { Maintenance } \\
\quad \text { (setting-level only) }\end{array}$} & $\begin{array}{l}\text { Assess outcomes } \geq 6 \\
\text { months }\end{array}$ & Individual long-term effects of the program & Interviews \\
\hline & Sustainability of program & Opportunities and challenges for program sustainability? & Interviews \\
\hline & $\begin{array}{l}\text { Measure of cost of } \\
\text { maintenance }\end{array}$ & What is the cost to maintain a program? & Interviews \\
\hline
\end{tabular}

* RE-AIM indicates Reach, Effectiveness, Adoption, Implementation, and Maintenance; F/R, free or reduced-price school lunch program; and HEI, Healthy Eating Index.

$\dagger \mathrm{HEI}-2010^{14}$ measures compliance with the 2010 Dietary Guidelines for Americans. HEI-2010 uses standards that are expressed as either a percentage of calories or per 1000 calories, separating diet quality from quantity. The sum of the scores for the food components is the total HEI-2010 score, which ranges from 0 to 100 , with a higher score indicative of a more healthful diet.

\section{PARTICIPANTS}

In order to gain a collective and statewide perspective of the program, the researchers utilized two purposive sampling strategies identified by Patton. ${ }^{22}$ These included maximum variation sampling, where participants were invited based on an array of positions, and snowball sampling, where additional participants were identified by study participants and then interviewed by the lead author to better understand phenomena within this program. The researchers sampled for participants until at least three individuals from an individual program (total programs across Montana $[\mathrm{N}=5])$ consented to contribute data to the statewide perspective. In addition, researchers recruited individuals with a state policy perspective about child hunger and backpack programs only and individuals with a state policy perspective and local involvement in backpack programs combined. This approach ensured triangulation of sources from people with different positions in the system and therefore was able to provide varied viewpoints. ${ }^{22}$

\section{METHOD}

The semistructured interview script was developed to align with Glasgow and colleagues' 21-item measure. ${ }^{12}$ Interview questions are included in the
"Measure" portion of Table 1. Interviews were deidentified by replacing each participant's name with a code number for anonymity and transcribed verbatim using Microsoft Word and Excel (v. 2008, Microsoft Corporation, Redmond, Washington). The deidentification process gave authors the ability to interpret meaning from a broad perspective of stakeholders across the state and gave participants the confidence to report personal perspectives without potential identification. In addition, although some of the participants responded using examples from their individual program perspective, using the described methodology below to analyze the Montana backpack program's collective scope of 
success and sustainability increased the ability to interpret meaning from multiple perspectives across the state. Authors were sure to include the diversity of all emerging themes from individual and statewide perspectives in the interpretation of data. Responses were not analyzed by position or relationship to individual backpack programs to ensure anonymity and garner a comprehensive perspective at the stakeholder level (i.e., across various positions rather than by various positions). Before conducting the study, the lead author discussed protocol with select stakeholders, and it was apparent that anonymity was paramount to collecting authentic perspectives about Montana backpack programs in this study.

Meaning units (MUs), or distinct data that constitute a single idea, were separated into distinct fragments. The two authors independently coded the transcripts based on deductive approach using the predetermined REAIM themes. The authors, both female doctorates with extensive training and experience in qualitative research methods, then independently coded for appropriate categories within each RE-AIM dimension.

To establish interrater reliability, authors met to confirm meaning or resolve discrepancies as needed. For example, the authors divided categories (e.g., separating "policy" from "proposed innovation") or collapsed categories (e.g., "program reach," "program expansion," and "program growth" included in "expansion of program") where appropriate. When the codes were divergent in meaning $(<15 \%$ of the time), the authors revisited the MU within the transcript to collaboratively decide the primary significance of the content.

The number of interviewees that reported MUs within a given category was also calculated to demonstrate data saturation within a theme and RE-AIM dimension (see "Category Name," "Interviewees," and "Meaning Units" columns in Tables 2 through 4). This provided data on the saturation of each category across the interviewees. Sample quotes are also offered in Tables 2 through 4 to demonstrate data authenticity. The lead author contacted participants to review their own transcript and results in order to member check the study interpretation. Approval was obtained from Montana State University Institutional Review Board for all components of this study with informed consent obtained from all participants.

\section{RESULTS}

The lead author conducted one-onone, semistructured interviews with 20 key informants. The interviews were conducted on the phone, with only the interviewee and interviewer on the line and in the room. The interviews lasted approximately 24 minutes ( $\pm 3.2 \mathrm{~min}$ utes). Interviewees were from state agencies $(n=2)$, food bank directors/ staff $(n=5)$, policy makers $(n=2)$, from school food service $(n=2)$, teachers $(n=2)$, parents $(n=1)$, registered dietitians $(n=1)$, from nomprofits invested in child well-being $(\mathrm{n}=2)$, from public health research $(\mathrm{n}$ $=1$ ), national level program informants $(n=2)$, principals $(n=2)$, and superintendents $(n=3)$. Five individuals declined to participate; their professional roles included superintendent $(n=2)$, policy maker, state agency director, and principal. Each individual declined because they did not see their knowledge about backpack programs as informative and then provided contact information for a more knowledgeable key informant. Of participants, 11 (55\%) were directly involved with the design and/or implementation of a local backpack program only (program $1[\mathrm{n}=2]$,

program $2[\mathrm{n}=3]$, program $3[\mathrm{n}=2]$, program $4[\mathrm{n}=2]$, and program $5[\mathrm{n}=$ 2]), 4 (20\%) participants were involved with backpack programs at the policy level in the state only, and $5(25 \%)$ participants were involved with a backpack program at the local level and at the policy level in the state (program 1 $[\mathrm{n}=1]$, program $3[\mathrm{n}=1]$, program 4 $[\mathrm{n}=2]$, and program $5[\mathrm{n}=1])$. Participants represented $100 \%$ of the backpack programs in Montana. Participants had various levels of knowledge about local, state, and larger policy level issues regarding backpack programs, food security, and child nutrition. In total, 387 MUs were identified across the 20 interviews.

\section{Reach}

Reach was estimated from a quantitative perspective. Because of program anonymity, reach was calculated based on the number of children participating in Montana backpack programs ( $\mathrm{n}$ $=2888$ ) divided by the number of children eligible for $\mathrm{F} / \mathrm{R}$ lunch across participating schools $(\mathrm{n}=12,465)$.

Each Montana backpack program reported the number of children participating on a weekly basis. F/R lunch participation was calculated using public data provided by the Montana Office of Public Instruction by school. This resulted in a participation rate of $23 \%$.

Six categories emerged from the qualitative interviews (see Table 2) around the dimension of reach: eligibility criteria ( $\mathrm{n}=17 \mathrm{MUs}$ ), expansion $(\mathrm{n}=15 \mathrm{MUs})$, high reach $(\mathrm{n}=4 \mathrm{MUs})$, sharing food ( $\mathrm{n}=4 \mathrm{MUs})$, stigma $(\mathrm{n}=$ 11 MUs), and supplemental $(\mathrm{n}=10$ MUs). The first emergent category within reach was the eligibility criteria related to recruiting children into the backpack programs. Some programs required parents to submit paperwork for their child to join the program: "The first year we did it, it was an optin program. We sent information to parents about the program. We told them that if their children need supplemental food, call the office, and sign the bottom of the paper." Other programs used an opt-out option: "We start the year with all of the children who are getting free and reduced meals. We put information in the backpack that if their child doesn't need the program to call the main office and they would be taken off of the list." There were mixed opinions about whether the program reached a representative proportion of children in need or if the program was still missing some of those who "need it most" because of eligibility criteria such as filing paperwork. The expansion category referred to the key informants' suggestions to expand the reach as well as current barriers to expansion, such as, "It's pretty much a private program, which can have its benefits, but it's not a universally available program." Four informants provided feedback that the program had a high reach because it assisted several children in schools across 
Table 2

Qualitative and Quantitative Results for Reach and Effectiveness Dimensions in Montana Backpack Program Evaluation During 2011-2012 School Year*

\begin{tabular}{|c|c|c|c|c|c|}
\hline Dimension & Category Name & $\begin{array}{l}\text { Interviewees, } \\
\text { No. }\end{array}$ & $\begin{array}{l}\text { Meaning } \\
\text { Units, No. }\end{array}$ & Example Meaning Unit & Quantitative \\
\hline \multirow[t]{6}{*}{ Reach } & Eligibility criteria & 9 & 17 & $\begin{array}{l}\text { "I look through my F/R list and sent the letter home to } \\
\text { families that I knew were homeless or in F/R lunch } \\
\text { program." }\end{array}$ & \multirow{6}{*}{$\begin{array}{l}23.2 \% \text { of children } \\
\text { participating who } \\
\text { are eligible to } \\
\text { participate† } \\
\text { across } 72 \\
\text { participating } \\
\text { schools }\end{array}$} \\
\hline & Expansion & 9 & 15 & $\begin{array}{l}\text { "Efficient and well-designed public relations campaigns } \\
\text { would actually make a huge difference." }\end{array}$ & \\
\hline & High reach & 4 & 4 & "We fed 520 very low-income, Title 1 students that year." & \\
\hline & Sharing food & 3 & 4 & $\begin{array}{l}\text { "We hear about some kids sharing food. It's not the intent } \\
\text { of the program, but we can't tell the kid not to, } \\
\text { obviously." }\end{array}$ & \\
\hline & Stigma & 5 & 11 & $\begin{array}{l}\text { "The social stigma of being a backpack kid. The kid who } \\
\text { doesn't have enough food at home." }\end{array}$ & \\
\hline & Supplemental & 6 & 10 & $\begin{array}{l}\text { "We send outreach materials for families to apply for } \\
\text { SNAP, WIC, and Summer Meals." }\end{array}$ & \\
\hline \multirow[t]{5}{*}{ Effectiveness } & $\begin{array}{l}\text { Comprehensive } \\
\text { solutions }\end{array}$ & 7 & 10 & $\begin{array}{l}\text { "Backpacks alone don't meet the need but there are other } \\
\text { resources that hopefully the family participates in." }\end{array}$ & \multirow{5}{*}{$\begin{array}{l}>50 \% \text { of students } \\
\text { eligible for free or } \\
\text { reduced lunch or } \\
\text { special } \\
\text { consideration } \\
\text { based on need }\end{array}$} \\
\hline & $\begin{array}{l}\text { Need to satiate } \\
\text { hunger }\end{array}$ & 15 & 35 & $\begin{array}{l}\text { "The backpack only puts a Band-Aid on a gaping wound. } \\
\text { It's not really addressing the full hunger need and the } \\
\text { larger issue." }\end{array}$ & \\
\hline & Pack content & 7 & 11 & $\begin{array}{l}\text { "They need to be able to prepare and eat the foods by } \\
\text { themselves." }\end{array}$ & \\
\hline & Positive perceptions & 14 & 36 & $\begin{array}{l}\text { "The kids feel better on Mondays and aren't afraid to go } \\
\text { home on Friday." }\end{array}$ & \\
\hline & $\begin{array}{l}\text { Unknown } \\
\text { effectiveness }\end{array}$ & 7 & 8 & $\begin{array}{l}\text { "We have not done any studies on [how far the food } \\
\text { stretches or meets the child's needs]." }\end{array}$ & \\
\hline
\end{tabular}

${ }^{*}$ F/R indicates free or reduced-price school lunch program; SNAP, Supplemental Nutrition Assistance Program; and WIC, Special Supplemental Nutrition Assistance Program for Women, Infants, and Children.

$\dagger$ Calculated based on number of children participating in backpack programs weekly divided by number of children who are F/R eligible.

Montana. One barrier to reach was the stigma associated with participation in a hunger relief program: "You do run into some issues with parents around pride. They don't want their children to be seen in need." Finally, the supplemental category was operationalized as the informants' perception that backpack programs were intended as supplemental to other hunger relief efforts across the state.

\section{Effectiveness}

Food bags scored an average HEI2010 score of $58.7 \pm 15.6(0$ [minimum score] to 100 [maximum score]; range $41.9-75.1) .^{13}$

Qualitative information about attrition was measured by seeking data about program dropout rates and reasons for dropout. Causes for program attrition included moving schools or the parent's no longer needing additional food resources. Key informants provided 100 MUs about the effectiveness of backpack programs across five categories; see Table 2 . The categories were comprehensive solutions ( $\mathrm{n}=10 \mathrm{MUs})$, need to satiate hunger ( $\mathrm{n}=35 \mathrm{MUs})$, pack content ( $\mathrm{n}$ $=11$ MUs), positive perceptions $(n=$ $36 \mathrm{MUs}$ ), and unknown effectiveness ( $\mathrm{n}=8 \mathrm{MUs}$ ). Unknown effectiveness related to informants' opinion that they either only have "hypothetical answers" or that "the numbers [they] get don't tell the whole story" about the program's ability to relieve child hunger over the weekend. On the other hand, 14 informants provided 36 MUs about positive perceptions of the program, from "students learn and behave better after receiving the packs" to "the kids love and consume it all." Informants also provided mixed opinions about the pack content's nutritional value and ease of use. Yet there was consistent reporting (i.e., 35 MUs across 15 informants) that there is still a need to satiate hunger on a larger scale. The consensus was that backpack programs "are not filling the true nutrition gap, but it really is helping the recipients." Informants also spoke about needing to combine efforts with other entities for more comprehensive solutions.

\section{Adoption}

There were 270 schools in Montana with $>50 \%$ children eligible for the $\mathrm{F} /$ $\mathrm{R}$ lunch program in 2011-2012. This is the denominator for potential schools to deliver backpack programs. In total, five food banks facilitated backpack program delivery at 70 schools during 2011-2012. Therefore, the adoption rate is $26 \%$. The Figure provides a map of Montana indicating where programs are being implemented and proximity to food banks. In general, most backpack programs were implemented in the western half of Montana. Participating schools were an average of 120 


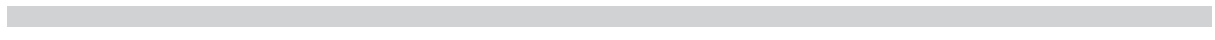

Figure

Map of Montana Backpack Programs and Food Bank Locations

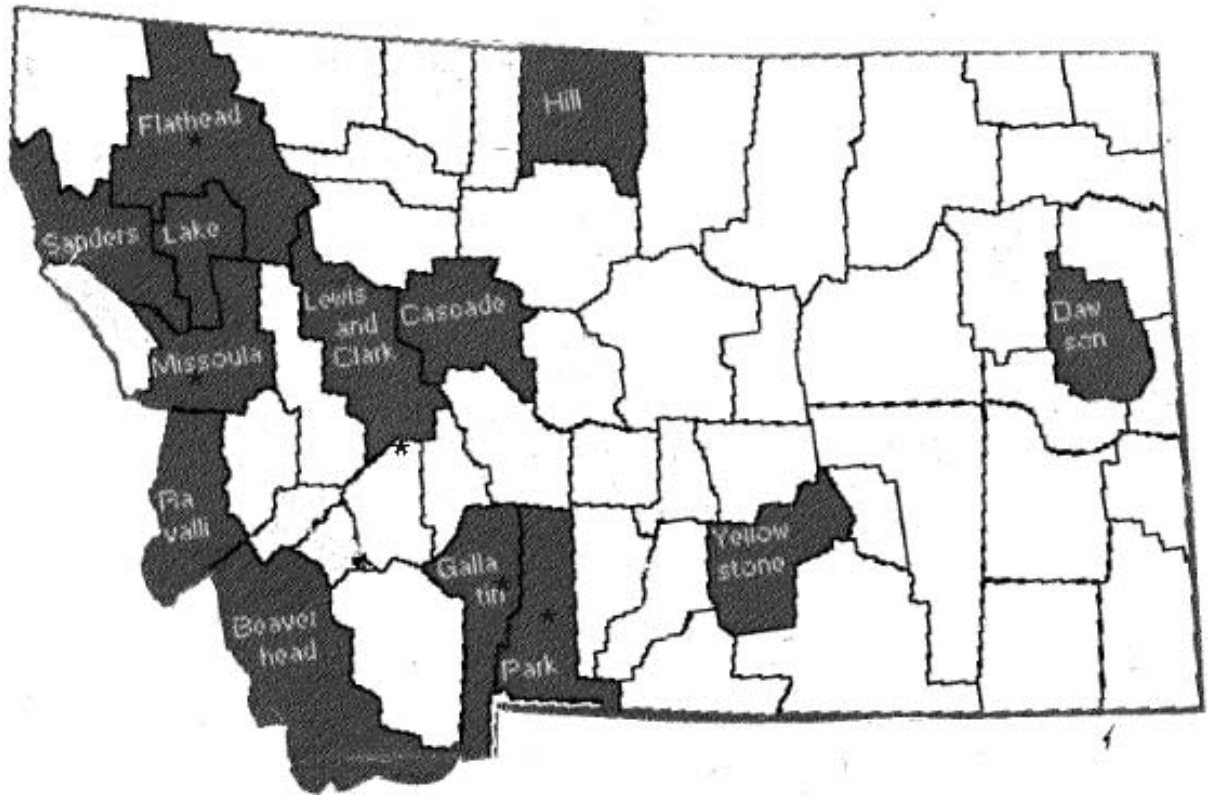

Darkened color denotes at least one backpack program within county. Asterisk denotes food bank location.

miles (range $=1-568$ miles) from the food bank facilitating the backpack program.

Qualitative adoption questions asked about the representativeness of settings and intervention agents who are willing to initiate the program. Setting-level results are seen in Table 3. Overall, seven categories emerged from the qualitative interviews that represent opportunities for and barriers to adoption: community involvement ( $\mathrm{n}=4$ MUs), evaluation $(\mathrm{n}=4$ MUs), expansion of program $(n=21$ MUs), financial constraints $(n=7$ MUs), food needs ( $n=10$ MUs), role clarity and acceptance ( $\mathrm{n}=15 \mathrm{MUs})$, and school eligibility ( $\mathrm{n}=17 \mathrm{MUs})$. School eligibility occurred when funding and resources (e.g., implementing agency, food, staff) were identified and based on need ( $>50 \%$ students eligible for $\mathrm{F} / \mathrm{R}$ lunch or special consideration). If funding and resources were not available or no need was identified, a school was excluded from participating. Staff who implemented the programs were invested in working to end childhood hunger. For role clarity and acceptance, interviewees noted that one salaried individual at the food bank and several volunteers typically operated the program and at least one school staff contact was necessary for helping to facilitate logistics and garnering school support. The level of expertise required for the program was based on navigating appropriate operating procedures for each site, which include garnering community involvement, overcoming financial constraints, expanding the program sustainably, appropriately satisfying food needs, and utilizing evaluation tools.

\section{Implementation}

Backpack programs $(\mathrm{n}=5)$ deliver bags to students $(\mathrm{N}=2889)$ in each school $(\mathrm{n}=70)$ every Thursday or Friday during the school year (September/August-May/June). Bags are distributed confidentially and into children's backpacks or lockers. The individual who delivers the bag is aware of this protocol and ensures appropriate bag delivery. However, if a student is not present on a particular Thursday or Friday, a bag is not distributed to that particular student. To measure implementation costs, we collected data and calculated the average cost per bag. Across the 70 schools, bags averaged \$3.87 (SD \pm .94$)$. Schools whose bags were less costly found ways to purchase food items in bulk and increase food donations.

Qualitative questions about implementation inquired about protocolspecifically the consistency of delivery, time, and cost of backpack programs. Seven categories emerged: adaptations ( $\mathrm{n}=8 \mathrm{MUs})$, bag contents $(\mathrm{n}=7 \mathrm{MUs})$, challenges ( $n=7$ MUs), costs $(n=7$ MUs), delivery systems ( $n=9$ MUs), fidelity ( $\mathrm{n}=7 \mathrm{MUs}$ ), and privacy $(\mathrm{n}=5$ MUs). Each key informant described a similar delivery system with protocol variations to fit the needs of each site, including where the bags are placed (e.g., in the child's backpack or locker), strategies for maintaining confidentiality, the day the foods are delivered (e.g., Thursday or Friday), who identifies children to participate, and what type of volunteers pack and distribute bags. The delivery systems are set up individually because "the program needs to be essentially seamless for the school." Interviewees indicated that several adaptations were made to similar backpack programs from the original protocol of the national BackPack Program. Adaptations included trying new and different bag contents (e.g., the addition of fresh produce), distributing bags during the summertime, sourcing foods at wholesale cost, and making special considerations for schools with $<50 \%$ of students eligible for $F / R$ lunch. Privacy to maintain the child's confidentially was key to program implementation. Challenges to implementation were based on logistics and included a flexible protocol, providing a variety of foods each week, transportation of foods from the food bank to the school, consistent funding and resources (e.g., volunteers and time), finding storage space for bags, and increasing school enrollment. The bag contents varied within each protocol. Key informants reported that including nutritious foods was important, but sometimes the need for kid-friendly, shelf-stable, and easy-to-prepare foods limited the health of foods. "We make nutritious 
Table 3

Qualitative Results for Adoption and Implementation Dimensions in Montana Backpack Program Evaluation During $2011-2012$ School Year*

\begin{tabular}{|c|c|c|c|c|}
\hline Dimension & Category Name & $\begin{array}{l}\text { Interviewees, } \\
\text { No. }\end{array}$ & $\begin{array}{l}\text { Meaning } \\
\text { Units, No. }\end{array}$ & Example Meaning Unit \\
\hline \multirow[t]{7}{*}{ Adoption } & $\begin{array}{l}\text { Community } \\
\text { involvement }\end{array}$ & 2 & 4 & "The local food banks work with a lot of the programs or hospitals." \\
\hline & Evaluation & 3 & 4 & "...evaluations, challenges, and what works." \\
\hline & Expansion of program & 10 & 21 & "There is certainly room to add to the number of schools." \\
\hline & Financial constraint & 5 & 7 & $\begin{array}{l}\text { "It's a difficult balance because there are more schools that want the } \\
\text { program than don't. The money is an issue with that." }\end{array}$ \\
\hline & Food needs & 7 & 10 & $\begin{array}{l}\text { "We are very stretched for food resources already in eastern Montana } \\
\text { with the energy boom." }\end{array}$ \\
\hline & $\begin{array}{l}\text { Role clarity and } \\
\text { acceptance }\end{array}$ & 10 & 15 & $\begin{array}{l}\text { "Definitely the workload. Is it added onto someone's position? ... } \\
\text { Sometimes it takes a while to actually figure out who will do it." }\end{array}$ \\
\hline & School eligibility & 12 & 17 & $\begin{array}{l}\text { "We do try to go to the schools that are in the highest need first or the } \\
\text { most eligible for F/R." }\end{array}$ \\
\hline \multirow[t]{7}{*}{ Implementation } & Adaptation & 13 & 8 & $\begin{array}{l}\text { "The model looks different at every food bank. We encourage that } \\
\text { because every community is different." }\end{array}$ \\
\hline & Bag contents & 15 & 7 & $\begin{array}{l}\text { "Backpack meals come in single servings. That is really important so } \\
\text { the food is ready to eat and the kids don't have to prepare." }\end{array}$ \\
\hline & Challenges & 16 & 7 & $\begin{array}{l}\text { "Other challenges are mostly logistical—distributing food, especially } \\
\text { perishable, transportation costs, constant funding and resources." }\end{array}$ \\
\hline & Cost & 16 & 7 & $\begin{array}{l}\text { "We average about } \$ 3 \text { per bag ... times } 230 \text { bags, that is } \$ 690 \ldots \text { by } \\
40 \text { school weeks and your budget starts going up fast." }\end{array}$ \\
\hline & Delivery systems & 20 & 9 & $\begin{array}{l}\text { "That same person lives near the food bank, picks it up, brings it to } \\
\text { school, and distributes to the person's locker on Fridays." }\end{array}$ \\
\hline & Fidelity & 10 & 7 & $\begin{array}{l}\text { "We've created materials based off of this about how to start a } \\
\text { backpack program, the best way for the teachers to identify high-risk } \\
\text { kids, and all of the resources, research, and materials from Feeding } \\
\text { America, and } 150 \text { other food banks' experiences around the nation." }\end{array}$ \\
\hline & Privacy & 15 & 5 & $\begin{array}{l}\text { "Our school staff does a great job in discreetly getting these bags out } \\
\text { to kids. I applaud them for their efforts." }\end{array}$ \\
\hline
\end{tabular}

${ }^{*} \mathrm{~F} / \mathrm{R}$ indicates free or reduced-price school lunch program.

food choices each week ... we get low sodium, whole grain, and low fat whenever we can. But there aren't a whole lot of choices in the single servings." Agencies received monetary and resource support to supplement the costs of implementing backpack programs, including from community organizations, food drives, and the state food bank network. This support to cover costs helped some agencies fulfill food needs for bags $100 \%$ of the time. Several strategies have been employed to encourage fidelity to backpack program protocol, including documenting program protocol (i.e., how to find funding and resources, how to purchase foods) as well as reporting why some sites succeed more than others.

\section{Maintenance}

At the time of the study (summer 2012), backpack programs reported being in operation for an average of 2.8 years (range $=2-4$ years).

Questions related to program maintenance were designed to assess outcomes, program sustainability, and cost of long-term program delivery. Overall, 10 categories emerged from the qualitative interviews around program maintenance: barriers ( $\mathrm{n}=4 \mathrm{MUs})$, collaboration, ( $\mathrm{n}=4 \mathrm{MUs})$, community involvement ( $\mathrm{n}=17 \mathrm{MUs}$ ), evaluation ( $\mathrm{n}=2$ MUs), funding $(\mathrm{n}=37$ MUs), innovation ( $\mathrm{n}=6 \mathrm{MUs})$, longterm maintenance $(n=8$ MUs), partners $(\mathrm{n}=7$ MUs), policy $(\mathrm{n}=6$ MUs), proposed innovations $(n=6$ MUs), and sustainable expansion $(\mathrm{n}=$ 7 MUs). Results for the maintenance dimension are reported in Table 4.
The single barrier reported to program maintenance was the lack of funding for resources (e.g., staff and time). Ideas to overcome this barrier included partners and community involvement. Interviewees echoed that a mix of public and private support for the program would provide for program maintenance, but that community support (especially through funding) was especially important. Similarly, key informants recognized the need for community involvement through fundraising, volunteer time, food donations or drives, and overall buy-in. Key informants noted that informal program evaluations were completed to help identify and decrease barriers to maintenance. Key informants contributed differing thoughts about funding for program maintenance. Some key informants conveyed that 


\section{Table 4}

Qualitative Results for Maintenance Dimension in Montana Backpack Program Evaluation During 2011-2012 School Year

\begin{tabular}{|c|c|c|c|c|}
\hline Dimension & Category Name & $\begin{array}{l}\text { Interviewees, } \\
\text { No. }\end{array}$ & $\begin{array}{l}\text { Meaning } \\
\text { Units, No. }\end{array}$ & Example Meaning Unit \\
\hline \multirow[t]{10}{*}{ Maintenance } & $\begin{array}{l}\text { Barrier for } \\
\text { maintenance }\end{array}$ & 3 & 4 & $\begin{array}{l}\text { "I think that one of the challenges is that a lot of the local communities don't } \\
\text { necessarily have the extra resources-especially ones with the most need." }\end{array}$ \\
\hline & Collaboration & 4 & 4 & $\begin{array}{l}\text { "A pairing of reimbursable component and community support would make the } \\
\text { program sustainable in the long term." }\end{array}$ \\
\hline & $\begin{array}{l}\text { Community } \\
\text { involvement }\end{array}$ & 9 & 17 & $\begin{array}{l}\text { "I really see it as a new aim for our community to see what teachers are dealing } \\
\text { with every day." }\end{array}$ \\
\hline & Evaluation & 2 & 2 & $\begin{array}{l}\text { "It gives you fresh ideas for building and obstacles. Also a comparison for how } \\
\text { quickly our program is growing." }\end{array}$ \\
\hline & Funding & 14 & 37 & $\begin{array}{l}\text { "We want to generate enough funds to expand the program to communities that } \\
\text { need it." }\end{array}$ \\
\hline & Innovation & 3 & 6 & "We have schools planting school gardens. " \\
\hline & $\begin{array}{l}\text { Long-term } \\
\text { maintenance }\end{array}$ & 5 & 8 & "We also need permanent solutions." \\
\hline & Policy & 7 & 7 & "The legislature really needs to support efforts to decrease childhood hunger." \\
\hline & $\begin{array}{l}\text { Proposed } \\
\text { innovation }\end{array}$ & 4 & 6 & $\begin{array}{l}\text { "If we could add nutrition components and education that would be a good } \\
\text { thing." }\end{array}$ \\
\hline & $\begin{array}{l}\text { Sustainable } \\
\text { expansion }\end{array}$ & 3 & 7 & "I am not willing to expand the program unless it can be sustainable." \\
\hline
\end{tabular}

partial state or federal funds would assist in program maintenance, whereas others held that funding should be generated from the community. Funding was noted as an essential part to sustainability: "The most obvious [challenge to sustainability] is finding a steady source of income for the program." Key informants noted that policy support for community-based backpack programs was challenging because of the cumbersome legislative process, lack of awareness about hunger in the public, and ideologies about supplemental programs. For long-term maintenance, interviewees recommended building self-sustaining programs. Several innovations have occurred to build self-sustaining programs: food pantries in schools, school gardens, and expanding the program to family members. Key informants also proposed innovations that included increased feedback from parents, expanding fundraising events, adding new foods, and starting a public relations campaign to build support. Lastly, sustainable expansion was regarded as important to program maintenance: "We have to ensure all of our programs can survive in the future and expand."

\section{CONCLUSION}

This is the first investigation, to our knowledge, that has used a mixedmethods approach to evaluate an ongoing community-based backpack program through the RE-AIM framework. ${ }^{23}$ Our preliminary results indicate that backpack programs aimed at providing weekend food assistance (1) are successful at reaching a proportion of representative children (reach), (2) are effective at providing food bags of modest dietary quality based on HEI2010 (effectiveness), (3) have large community adoption and support, with a pragmatic level of expertise required to deliver the program (adoption), (4) have protocol fidelity that is relatively easy to follow (implementation), and (5) have had adaptations (e.g. food donations), that increase the potential program sustainability across Montana (maintenance).

The overall intent of the study was to gather information that aligned with each RE-AIM dimension in order to enhance the inferences drawn related to the backpack program. Identifying factors that may enhance each dimensions of RE-AIM may generate largerscale adoption and promotion (e.g., statewide backpack programs). Many systematic reviews of health promotion programs (using the RE-AIM framework) have found that issues associated with adoption and maintenance are drastically underrepresented in the literature. $^{24,25}$ That is, the indicators associated with the adoption and maintenance dimensions (see Table 1, "Items") are the least reported across the five dimensions. This study found resources and funding as important barriers to adoption and maintenance. Notably, the full support of adopting agents directly influences implementation fidelity and program sustainability. ${ }^{26}$ Our results indicate that program adopters explore several program adaptations (e.g., fundraising, collaborating with partners) in order to promote sustainability. Most important, to promote long-term sustainability of backpack programs, communities must explore solutions to sustainable funding.

More nuanced findings suggest that those who are involved in individual and school identification, food procurement, and delivery procedures support the mission and values of backpack programs. Key informants also felt the program was effective in providing supplemental food relief and also assisted in decreasing the 
negative effects of hunger (e.g., emotional, behavioral). Some salient concerns were around continued support (i.e., financially) from entities inside and outside of the community. Key informants were consistent with feed-

back about the ease of delivery and the importance of discretion to avoid stigma. Concerns were also raised about the nutritional value of the backpack contents, and the HEI-2010 analysis validated these concerns. The average HEI-2010 score of 58.7 is within the range of the average dietary quality score for the American child (54.7 and 59.6) ${ }^{27}$ Finding foods that were high in nutrient quality, affordable, shelf stable, and easy to prepare provided a challenge to increased nutrient quality of bag contents.

A key strength of this study was the use of the RE-AIM framework as a guiding metric for the evaluation of emergency weekend backpack food assistance programs. RE-AIM has previously been used to evaluate numerous health promotion programs, a peer leadership lifestyle intervention for adolescent girls, ${ }^{26}$ and weight loss interventions, ${ }^{25}$ yet the data collected often lack information on external validity factors. ${ }^{24}$ This study directly addresses the paucity in the literature related to the perspectives of adopters and implementers around sustainability and logistics of community-based program delivery. The evaluation methodology presented here can also be used to evaluate other health promotion programs, especially interventions that do not have direct measures of effectiveness. The authors were able to apply their extensive REAIM expertise in this study and reflected that the development of a REAIM-based interview guide may be helpful in other evaluation studies. The deductive approach (i.e., using each dimension of RE-AIM as a theme) allowed the authors to capture barriers and facilitators related to the success of the backpack program.

Some limitations of this study exist around the inability to gather primary data from program participants because of anonymity. Divergent perspectives from different participants' positions were also not able to be distinguished. From a RE-AIM perspective, we were unable to identify individually based effectiveness and behavior maintenance. However, this limitation is moderated because the current study evaluated backpack programs from a systematic and not an individual standpoint. Individual-level data (i.e., reach, effectiveness, maintenance) were collected and aggregated, when possible, to provide support for the system-level variables. Using a mixed-methods approach through an esteemed evaluation framework provides strength to this analysis. Specifically, Kessler et al. ${ }^{28}$ reemphasize the need to include qualitative measures to capture rich data regarding external validity factors. This study was designed with the specific intention of collecting data from key informants to assist in the translation of our findings; 20 interviewees contributed to the development of categories via saturated data points. Further, the use of RE-AIM assists in the ability to make national recommendations for emergency weekend backpack food assistance programs.

These mixed-methods results inform practice by acknowledging that the backpack programs in Montana are a valued part of the food assistance system for children and that there is room for improvement in the foods offered and expansion of the program to those in need. Facilitating venues to share information about best practices in food sourcing and distribution between backpack programs will increase knowledge about how to improve the program statewide. The data from key stakeholders represent an underlying notion that encouraging communities to take ownership (and to acknowledge that childhood hunger exists in their community) will help to promote growth and sustainability of the backpack program. The backpack programs are just one way for communities to provide families with support that includes alternative supplemental food resources. Other community-supported social services also exist to help families find solutions to food and resource shortages and ultimately decrease demand on the backpack program.

Findings from this study support that backpack programs are widespread and growing in Montana and are effective in relieving weekend hunger. Further- more, program findings were shared with stakeholders to validate responses and also serve as a platform for coordination and discussion in communities. This program is also run nationally, and it is important to consider its larger-scale implications for the 8.3 million children who struggle with hunger nationwide. ${ }^{1}$ Further research and evaluation efforts investigating how to best support backpack program adoption and maintenance from state to state are warranted (e.g., differences in rural versus urban settings). Ultimately, community-based hunger relief programs with a broad reach, evidenced effect, inviting adoption, and feasible implementation may perpetuate a system that will simultaneously alleviate immediate family food needs and promote long-term solutions to end hunger alongside public assistance programs.

\section{SO WHAT? Implications for Health Promotion Practitioners and Researchers}

What is already known on this topic?

Weekend backpack food assistance programs are implemented nationally and serve more than 8.3 million children struggling with hunger.

\section{What does this article add?}

Backpack programs are run nationally and it is important to consider their larger-scale implications. Our preliminary results indicate that backpack programs aimed at providing weekend food assistance (1) are successful at reaching a proportion of representative children (reach); (2) are effective at providing food bags of modest dietary quality based on HEI-2010 (effectiveness); (3) have large community adoption and support, with a pragmatic level of expertise required to deliver the program (adoption); (4) have protocol fidelity that is relatively easy to follow (implementation); and (5) have had adaptations (e.g. food donations) that increase the potential program sustainability across Montana (maintenance).

What are the implications for health promotion practice or research?

This is the first investigation, to our knowledge, that used a mixed-methods approach to evaluate an ongoing community-based backpack program through the RE-AIM framework. 


\section{References}

1. US Dept of Agriculture Economic Research Service. Food security in the US. Available at: http://www.ers.usda.gov/ topics/food-nutrition-assistance/foodsecurity-in-the-us / key-statistics-graphics. Accessed September 20, 2013.

2. Kilpatrick SI, McIntyre L, Potestio ML. Child hunger and long-term adverse consequences for health. Arch Pediatr Adolesc Med. 2010;164:754-762.

3. Melchior M, Caspi A, Howard L, et al. Mental health context of food insecurity: a representative cohort of families with young children. Pediatrics. 2009;124:e564e572.

4. Kleinman RE, Murphy JM, Little M, et al. Hunger in children in the United States: potential behavioral and emotional correlates. Pediatrics. 1998;101:E3.

5. US Dept of Agriculture, Food and Nutrition Service. Healthy Hunger-Free Kids Act of 2010. Available at: http://www. fns.usda.gov/cnd/governance/ legislation/cnr_2010.htm. Accessed September 20, 2013.

6. Guo B. Beyond the public safety net: the role of nonprofits in addressing material hardship of low-income households. Nonprofit Volunt Sect Q. 2010;39:784-801.

7. Cotunga N, Forbes S. A BackPack Program provides help for weekend child hunger. $J$ Hunger Environ Nutr. 2008;2:39-35.

8. Rogers Y, Milewska M. Food assistance through the school system: evaluation of the Food for Kids Backpack Program. Journal of Children and Poverty. 2007;13:7595.

9. Feeding America. BackPack Program. Available at: http://feedingamerica.org/ how-we-fight-hunger/programs-andservices/child-hunger/backpack-program. aspx. Accessed September 20, 2013.

10. Montana Food Bank Network. BackPack Program. Available at: http://mfbn.org/ learn/programs/backpack-program. Accessed September 20, 2013.

11. Glasgow RE, Vogt TM, Boles SM. Evaluating the public health impact of health promotion interventions: the REAIM framework. Am J Public Health. 1999; 189:1322-1327.

12. Glasgow RE, Klesges LM, Dzewaltowski DA, et al. The future of health behavior change research: what is needed to improve translation of research into health promotion practice? Ann Behav Med. 2004;27:3-13.

13. Guenther PM, Casavale KO, Reedy J, et al. Update of the Healthy Eating Index2010. J Acad Nutr Diet. 2013;113:569-580.

14. Harnack L, Hearst M, Harrison M. Report to Feeding America: evaluation of the nutrition quality of BackPack Program menus; 2012. Available at: fromhungertohealth.files.wordpress.com/ 2012/05/evaluation-of-the-nutritionalquality-of-backpack-program-menus-finalreport.pdf. Accessed September 20, 2013.

15. Byker C, Smith T. Food assistance programs for children afford mixed dietary quality based on HEI-2010. Nutr Res. 2015;35:35-40.

16. Montana Office of Public Instruction. Free/reduced eligibility data. Available at: https://apps.opi.mt.gov/ opireportingcenter/frmPublicReports. aspx?ProcName=procPublicReporting FreeReducedSelect\&ScreenTitle=Free / Reduced\%20Eligibility\% 20Data\&SelectStateFy=1. Accessed March 28, 2015.

17. Montana Food Bank Network. Homepage. Available at: http://mfbn.org. Accessed September 20, 2013.

18. Gallatin Valley Food Bank. Homepage. Available at: http://www. gallatinvalleyfoodbank.org. Accessed September 20, 2013.
19. Helena Food Share. Homepage. Available at: http://www.helenafoodshare.org/ janda/index.php. Accessed September 20, 2013.

20. Livingston Food Pantry. Homepage. Available at: http://livingstonfoodpantry. org. Accessed September 20, 2013.

21. Flat Head Food Bank. Homepage. Available at: http://www. flatheadfoodbank.com. Accessed September 20, 2013.

22. Patton M. Qualitative Evaluation and Research Methods. Thousand Oaks, Calif: Sage; 1990:169-183.

23. RE-AIM. Homepage. Available at: http:// www.re-aim.org. Accessed January 22, 2014.

24. Gaglio B, Shoup J, Glasgow RE. The REAIM framework: a systematic review of use over time. Am J Public Health. 2013;103: e38-e46.

25. Akers J, Estabrooks PA, Davy BM. Translational research: bridging the gap between long-term weight loss maintenance research and practice. $\mathrm{J} \mathrm{Am}$ Diet Assoc. 2010;110:1511-1522.

26. Jenkinson KA, Naughton G, Benson AC. The GLAMA (Girls! Lead! Achieve! Mentor! Activate!) physical activity and peer leadership intervention pilot project: a process evaluation using the RE-AIM framework. BMC Public Health. 2012;12:55.

27. Fungwe T, Guenther P, Juan W, et al. The quality of children's diets in 2003-04 as measured by the Healthy Eating Index 2005. Alexandria, Va: Center for Nutrition Policy and Promotion, US Dept of Agriculture; 2009. Available at: www.cnpp. usda.gov/Publications/NutritionInsights/ Insight43.pdf. Accessed September 20, 2013.

28. Kessler RS, Purcell EP, Glasgow RE, et al. What does it mean to "employ" the REAIM model? Eval Health Prof. 2013;36:4466. 